\title{
Cigarette smoking on the functional recovery of patients with rotator cuff tear submitted to physical therapy
}

\author{
Taís Cardoso Leffa \\ Jéssica Galvão Novelli \\ Gabrielle Biff dos Santos \\ Gizele Maciel Bello \\ Mariele da Silva Santos \\ Micaela Martins Silveira \\ Roberta C. Leindecker \\ Rodrigo Boff Daitx \\ Marcelo Baptista Dohnert
}

Physiotherapy Course, Lutheran University of Brazil, Torres/RS, Brazil

Corresponding author:

Marcelo Baptista Dohnert

Rua Gonçalves Chaves 3949

bloco A apto 201

Pelotas/RS, Brazil

Tel.: +53 984632952

E-mail: mdohnert@hotmail.com

\section{Summary}

Introduction: Rotator cuff (RC) tear is an inflammatory and degenerative shoulder disease. Nicotine is a potent vasoconstrictor that decreases oxygen support in the Codman's critical zone.

Methods: Nonrandomized clinical trial of 24 patients with $\mathrm{R} / \mathrm{CT}$ divided into nonsmoking $(\mathrm{n}=12)$ and smoking $(n=12)$ groups, which received physical therapy three times a week for four weeks. The following were evaluated: pain level, range of motion (ROM), muscle strength, and functionality. Results: Pain was reduced in the long term only in the nonsmoking group. Functionality was improved in the nonsmoking group. Smokers started the treatment with a lower ROM of forward flexion, abduction, and external rotation of the left shoulder. After the intervention, they remained with less forward flexion and abduction of the left shoulder $(p<0.05)$. The nonsmoking group increased the ROM of bilateral forward flexion, abduction, extension, and external rotation of the right upper limb $(p<0.05)$. In the smoking group, muscle strength was lower for forward flexion, abduction, bilateral external rotation of the shoulder, and internal rotation of the left shoulder after the interventions $(p<0.05)$.

Conclusion: Cigarette smoking worsens functional outcomes following a physical therapy program for shoulder RCT.

Level of evidence: Ilb.

KEY WORDS: shoulder impingement syndrome, smoking, tendinopathy.

\section{Introduction}

Shoulder pain is the third most common musculoskeletal condition, with rotator cuff tear (RCT) being more prevalent, affecting 18 to $31 \%$ of the general population monthly ${ }^{1}$. The prevalence of rotator cuff (CR) lesions and ruptures increases with age. At age of 50 , the prevalence is $10.7 \%$ and increases to $36.6 \%$ for people with 80 years or older ${ }^{1}$. Rotator cuff (RC) tears are of inflammatory and degenerative origin, characterized by mechanical impingement or compression of certain structures located in the subacromial space, especially the supraspinatus tendon, the long head of the biceps, the subacromial bursa, and the acromioclavicular joint ${ }^{2}$. Any anatomo-pathological alteration that changes the muscle synergy or compromises the normal biomechanics of the shoulder complex where the action of the deltoid prevails over the action of the RC will lead to the decrease of the subacromial space and the clamping of the subacromial space structures ${ }^{2}$.

Neer described three stages of the Impingement Syndrome $^{3}$. Stage I is characterized by edema, inflammation, and hemorrhage of the bursa and tendons of the $R^{3}$. Stage II comprises fibrosis and tendinitis ${ }^{3}$. Stage III represents the stage of RC rupture, linked to bone changes in the humeral head and acromion ${ }^{3}$.

Smoking is a major global health problem. Studies have shown the negative impacts of smoking on wound healing, fracture healing, and tissue repair, being also associated with the development of musculoskeletal injuries ${ }^{4-6}$.

Tendons are metabolically hypoactive tissues and require vascular support ${ }^{7}$. Following this theory, a tendon with poor vascular supply is the supraspinatus muscle tendon, located in an area called the Cod- 
man's critical zone ${ }^{7}$. Smokers are further impaired by the fact that nicotine and carbon monoxide produce vasoconstriction, decreasing tissue oxygen supply, thus contributing to degenerative tendon processes ${ }^{5,8,9}$.

There is a higher incidence of RC tear in smoking patients ${ }^{10}$, in addition to increased chances of RC rupture, and larger regions affected $6,8,11,12$. These patients also present worse surgical outcomes ${ }^{8,9,13}$.

There are few studies in the literature addressing the influence of smoking on the physical recovery of subjects with musculoskeletal injuries ${ }^{14}$. In postoperative outcomes of $\mathrm{RC}$ recovery, functional scores are shown to be lower in smokers, demonstrating that cigarette smoking interferes with the outcome of $\mathrm{RC}$ tear recovery ${ }^{14}$.

Thus, the purpose of this study was to determine whether there is or not a difference between the physical recovery from $\mathrm{RC}$ tear in smokers compared to nonsmokers.

\section{Material and methods}

\section{Study Design}

Nonrandomized clinical trial. The study was a collaboration of the Group of Studies and Research in Sports Rehabilitation and Orthopedic Trauma (GEPRETO) of the Lutheran University of Brazil, Torres/RS Campus, Brazil.

\section{Ethical Aspects}

The study was approved by the Ethics Committee of the Lutheran University of Brazil under number $1,340,814$. Study participants who met the eligibility criteria signed a consent form authorizing physical therapy treatment. This study meets the ethical standards of the Muscle, Ligaments and Tendons Journal ${ }^{15}$.

\section{Eligibility Criteria}

The eligibility criteria are shown in Table I.

\section{Evaluation protocol}

The evaluation protocol was performed on four occasions: initially; at the end of the intervention protocol; 90 days after the protocol; and six months after the protocol. Evaluations were performed by an independent (blind) evaluator.

\section{Pain level assessment}

Data regarding the assessment and measurement of pain were collected using visual analogue scale (VAS).

Assessment of cigarette smoking (Pack-Years) Pack-Years is a unit used to measure the number of cigarettes smoked by an individual over a long period of time. It is calculated by multiplying the number of packs of cigarettes smoked per day by the number of years the person smoked, or the number of cigarettes smoked per day is divided by 20 and then multiplied by the number of years smoked.

\section{Assessment of Range of Motion (ROM)}

Active and passive forward flexion, abduction, extension, internal and external rotation were measured using a universal goniometer.

\section{Assessment of muscle strength}

Data regarding the assessment of muscle strength were collected by manual dynamometry. The maximum voluntary isometric contraction (MVIC) of forward flexion, abduction, internal and external rotation was bilaterally evaluated. MVIC was measured at a mean angle of the arc of each movement.

\section{Assessment of functional level and quality of life} Functionality and disability data were collected using modified UCLA questionnaires on Functionality and Quality of Life, the Constant-Murley scale, and the Shoulder Pain and Disability Index (SPADI).

\section{Intervention protocol}

The intervention protocol consisted of closed kinetic chain (CKC) exercises, open kinetic chain (OKC) exercises, and scapulothoracic exercises. As the patients became familiar with the program and increased their mobility and strength, the difficulty and the load of each exercise were intensified.

The program lasted four weeks, with sessions three times a week (Tab. II).

Table I. Eligibility criteria.

\section{Inclusion Criteria}

Exclusion Criteria
- Patients with clinical diagnosis of rotator cuff injury classified as grade I and II RCl in the Neer scale, affected for at least three months, and aged between 18 and 70 years.

- Grade III RCI (Neer scale)

- Having two consecutive or three alternate absences in the treatments

- Patients who have undergone shoulder surgery or a rehabilitation program in the last three months

- Associated injuries such as: glenohumeral dislocation, calcareous tendonitis, LHB tendon injury, shoulder osteoarthritis, adhesive capsulitis, systemic or neurological disease

- Ethylism. 
Table II. Exercise Protocol.

\begin{tabular}{lr}
\hline \multicolumn{1}{c}{ Program } & No. series/repetitions \\
\hline FIRST WEEK & $3 \times 15$ repetitions \\
Exercise no. 1: Codman's pendular exercise & $3 \times 15$ repetitions \\
Exercise no. 2: Stick exercise & $3 \times 15$ repetitions \\
Exercise no. 3: Low row exercise & $3 \times 15$ repetitions \\
Exercise no. 4: External rotation with a stick & $3 \times 15$ repetitions \\
Exercise no. 5: Scapular stabilization & $3 \times 15$ repetitions \\
Exercise no. 6: "Sawing" &
\end{tabular}

\section{SECOND WEEK}

Exercise no. 1: Internal rotation with Theraband

$3 \times 15$ repetitions

Exercise no. 2: External rotation with Theraband

$3 \times 15$ repetitions

Exercise no. 3: Scapular waist exercise

$3 \times 15$ repetitions

Exercise no. 4: "Sawing" with Theraband

$3 \times 15$ repetitions

Exercise no. 5: Shoulder flexion with Theraband

$3 \times 15$ repetitions

Exercise no. 6: Abduction and elevation in CKC on the wall

$3 \times 15$ repetitions

\section{THIRD WEEK}

Exercise no. 1: Wall Push-up

$3 \times 15$ repetitions

Exercise no. 2: Trunk elevation with the arms

$3 \times 15$ repetitions

Exercise no. 3: Weight transfer

$3 \times 10$ seconds

Exercise no. 4: Mini bipodal squatting on unstable ground

$3 \times 15$ repetitions

Exercise no. 5: Scapular round-the-clock exercise

$3 \times 15$ repetitions

Exercise no. 6: Abduction and elevation in CKC on the wall

$3 \times 15$ repetitions

\section{FOURTH WEEK}

Exercise no. 1: Towel exercise

$3 \times 10$ repetitions

Exercise no. 2: Unipodal weight transfer

$3 \times 10$ seconds

Exercise no. 3: "Sawing" against gravity

$3 \times 15$ repetitions

Exercise no. 4: Scapular control

$3 \times 2$ repetitions for each sequential movement

Exercise no. 5: Unipodal squatting on a step

$3 \times 15$ repetitions

Exercise no. 6: Muscle reinforcement

$2 \times 10$ repetitions for each rotation 
At the end of each session during the four weeks, participants performed cryotherapy for 10 minutes.

\section{Sample calculation}

The primary endpoint of the study was the level of functionality, assessed through the UCLA scale score. Moreover, based on the study by Mallon et al. $(2004)^{16}$, we estimated the mean and standard deviation of the UCLA score of smokers participating in the study as $25.3 \pm 6.15$, and the mean and standard deviation of the UCLA score of nonsmokers as $32.01 \pm$ 3.76 after treatment. Therefore, an expected mean difference in UCLA scores of 6.71 was used between the groups, using a study power of $80 \%$, a significance level of $95 \%$, and a sample size ratio of $1: 8$ (smokers: nonsmokers), reaching the estimated number of eight smokers and eight nonsmokers, totaling 16 subjects. Believing that losses and refusals to be around $50 \%$, we reached the final number of 12 smokers and 12 nonsmokers, totaling 24 subjects.

\section{Statistical analysis}

The SPSS (Statistical Package for the Social Sciences) version 22.0 was used as statistical package. The study subjects were analyzed in the total sample and then stratified into a smoking group and a nonsmoking group. Data were initially analyzed descriptively and expressed as frequency, mean and standard deviation, and analyzed statistically by analysis of variance (ANOVA) for repeated measures of analysis, within each group, from the initial to the follow-up evaluation results in both smokers and nonsmokers. To compare the results between smokers and non- smokers, the unpaired Student's t-test was performed. The significance level established for the statistical test was $p<0.05$.

\section{Results}

Initially, 30 subjects with RC injury were selected. Four were excluded prior to the study for presenting grade III $\mathrm{RCl}$ according to the Neer classification. After the initial evaluation, two subjects were excluded by withdrawal. Therefore, the study initially had 24 subjects, 12 smokers and 12 nonsmokers. Of these, two smokers were not reevaluated at 90 and 180 days (6 months) due to other health problems (Fig. 1).

The mean age of the study subjects was $55.13 \pm$ 14.01 years. Fifteen $(62.5 \%)$ subjects were female. Eight were retired. Twenty-three $(95.8 \%)$ were white. The time of shoulder pain was $3.57 \pm 6.34$ years. Fourteen subjects $(58.3 \%)$ had right shoulder injury. The time of cigarette use was $30.67 \pm 12.19$ years. Cigarette consumption per day was $12.17 \pm 8.42$. Pack-Years was $20.21 \pm 17.49$. Smokers and nonsmokers were homogenous regarding the variables age, gender, occupation, skin color, time of pain, and affected shoulder ( $p>0.05$ ) (Tab. III).

A significant reduction of pain was observed in all study subjects immediately after the intervention protocol. However, in the 90- and 180-day evaluations, smokers again had pain levels similar to the baseline. The nonsmoking group reduced pain from $5.58 \pm 2.42$ points to $1.66 \pm 1.87$ points in the final evaluation $(p=0.001)$. In the 90-day evaluation, pain remained at

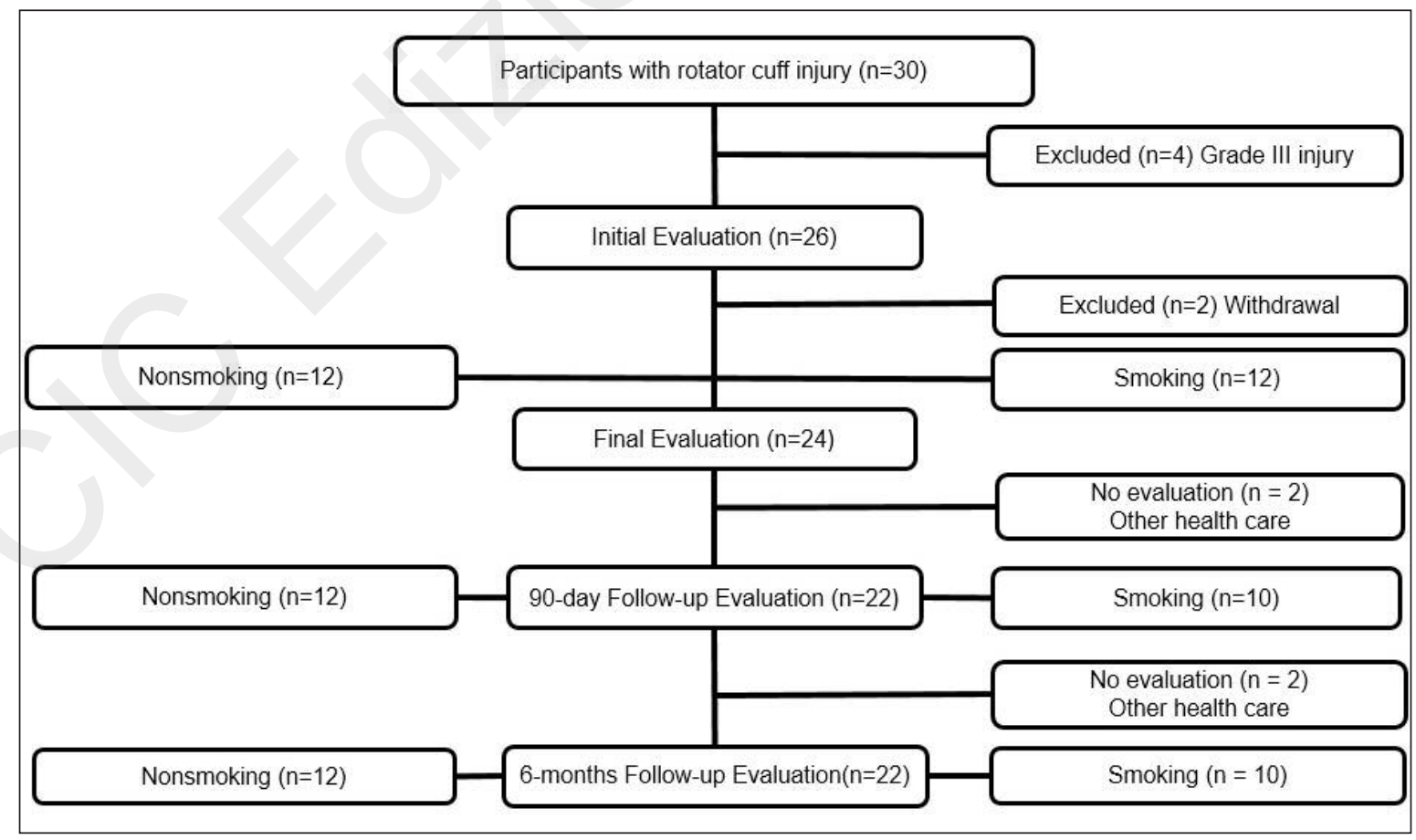

Figure 1. Study flow chart. 
Table III. Characterization of the study sample.

\begin{tabular}{|c|c|c|c|}
\hline Variable & Nonsmoking Group & Smoking Group & $p$ value \\
\hline Age, years $($ mean $\pm s d)$ & $58.63 \pm 10.01$ & $52.30 \pm 17.13$ & 0.28 \\
\hline Gender, M/F & $5 / 7$ & $4 / 8$ & 0.67 \\
\hline Occupation, n (\%) & & & 0.40 \\
\hline Retired & $5(41.5)$ & $3(24.9)$ & \\
\hline General Services & $0(0.0)$ & $2(16.6)$ & \\
\hline Homemaker & $2(16.6)$ & $2(16.6)$ & \\
\hline Others & $7(41.9)$ & $7(41.9)$ & \\
\hline Skin color, n (\%) & & & 0.31 \\
\hline White & $12(100.0)$ & $11(91.7)$ & \\
\hline Black & $0(0.0)$ & $1(8.3)$ & \\
\hline Time of pain, years (mean $\pm s d)$ & $3.71 \pm 8.46$ & $3.43 \pm 3.50$ & 0.39 \\
\hline Affected shoulder, n (\%) & & & 0.25 \\
\hline Right & $9(75.0)$ & $5(41.7)$ & \\
\hline Left & $1(8.3)$ & $3(25.0)$ & \\
\hline Both & $2(16.7)$ & $4(33.3)$ & \\
\hline Cigarette consumption, day (mean $\pm \mathrm{sd}$ ) & - & $12.17 \pm 8.42$ & - \\
\hline Pack-Years & - & $20.21 \pm 17.49$ & - \\
\hline $\begin{array}{l}\text { Time of cigarette consumption, years } \\
\text { (mean } \pm s d)\end{array}$ & - & $30.67 \pm 12.19$ & - \\
\hline
\end{tabular}

$2.0 \pm 2.33$ points $(p=0.005$ in relation to the initial evaluation), and six months after the intervention the pain value was $2.37 \pm 3.58$ points $(p=0.05$ compared to the initial evaluation). The smoking group, in turn, reduced pain from $5.91 \pm 2.31$ to $3.33 \pm 2.53$ points in the final evaluation $(p=0.05)$. Notwithstanding, in the 90-day follow-up, the pain level increased to $3.00 \pm$
3.09. In the six-month evaluation, this value was 4.50 \pm 3.37 (Fig. 2).

The level of functionality, assessed through the UCLA scale score, was significantly higher in nonsmokers in the final evaluation and in the 90-day reevaluation. Only the nonsmoking group presented a significant increase in the score at the end of the intervention,

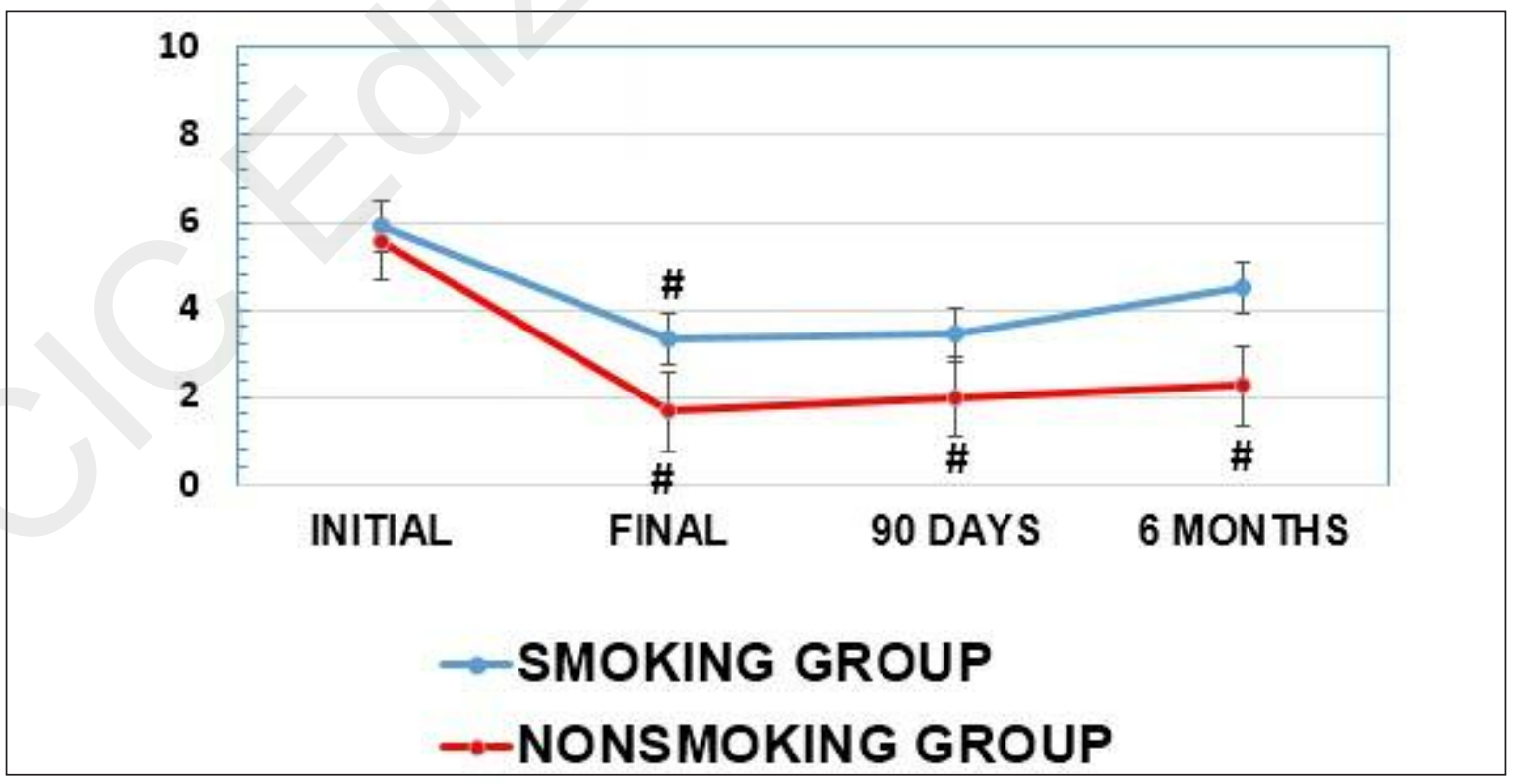

Figure 2. Pain level assessment (VAS) in the study groups.

Legend: \#, $p<0.05$ in relation to the initial assessment within the group. 
maintaining these scores in the 90-and 180-day evaluations. The initial score of nonsmokers was $20.67 \pm$ 5.61 points, increasing to $30.33 \pm 3.03$ points in the final evaluation $(p=0.001)$, with $29.67 \pm 5.19$ points in the 90-day evaluation ( $\mathrm{p}=0.0002)$, and remaining at $28.38 \pm 9.05$ points in the 180 -day evaluation $(0.02)$ (Fig. 3).

In the initial evaluation, $91.7 \%$ of smokers presented UCLA scale scores considered regular/poor, while in the nonsmoking group $75 \%$ were classified as regular/poor. Six months after the intervention protocol, $70 \%$ of smokers still had regular/poor results, whereas in the nonsmoking group only $16.6 \%$ had regular/poor results $(p<0.05)$ (Fig. 4).

The Constant-Murley score significantly improved in nonsmokers in all evaluations compared to the baseline. The initial score was $67.00 \pm 18.60$ points, increasing to $91.25 \pm 12.49$ points in the final reevaluation $(p=0.002)$. In the 90- and 180-day evaluations, the score remained at $88.08 \pm 16.50$ points $(p=0.006)$ and $88.08 \pm 24.69$ points $(p=0.05)$, respectively. The smoking group showed score improvement only in the 90-day evaluation compared to the baseline. The score of the smoking group was significantly lower than that of the nonsmoking group in the final reevaluation $(p=0.02)$ and at 180 days $(p=0.05)$ (Fig. 5).

The SPADI-Brazil questionnaire also showed a significant functional improvement in the nonsmoking group compared to the smoking group in the 180-day evaluation of the total SPADI score $(p=0.05)$ and in the subitem SPADI pain $(p=0.04)$. In the nonsmoking group, the initial SPADI pain score was $68.13 \pm$ 24.48 , decreasing to $23.13 \pm 17.33$ in the final evaluation $(p=0.000)$, and remaining at $23.67 \pm 23.31$ $(p=0.000)$ in the 90-day evaluation, and at $20.28 \pm$ 27.76 in the 180-day evaluation $(p=0.001)$. Regarding SPADI disability, the initial score was $45.55 \pm 28.26$, decreasing to $17.25 \pm 14.15$ in the final evaluation $(p=0.001)$, to $13.71 \pm 15.48$ in the 90 -day evaluation $(p=0.014)$, and to $11.58 \pm 23.04$ in the six-month evaluation $(p=0.011)$. Total SPADI significantly decreased from the initial evaluation to the other evaluations $(p<0.05)$. Nonetheless, the smoking group reduced the SPADI pain score only in the 90-day evaluation, but this score increased significantly in the sixmonth evaluation $(p<0.05)$. Regarding SPADI disabili-

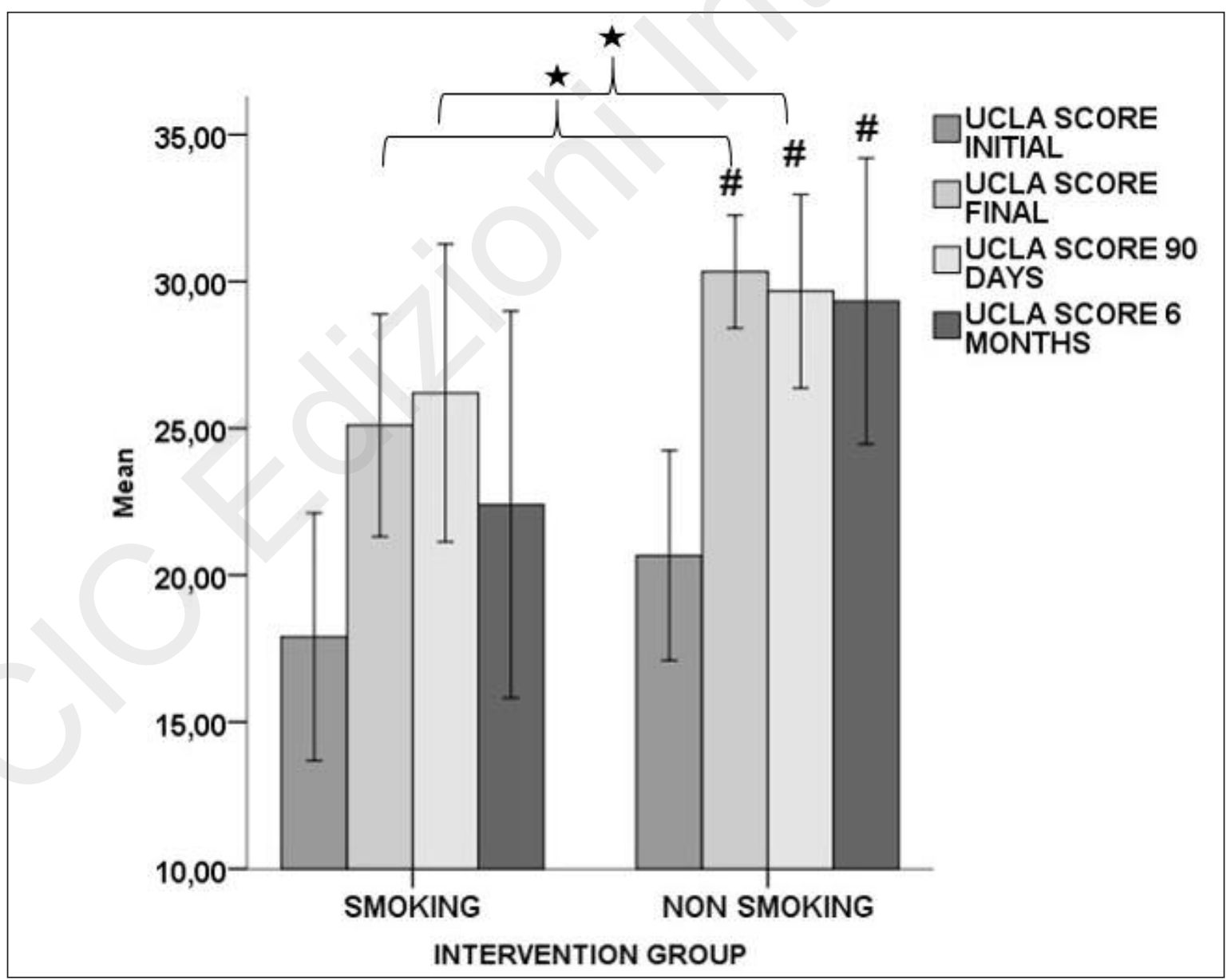

Figure 3. Score of the UCLA questionnaire in the study groups.

Legend: *, $p<0.05$ in relation to the same evaluation of the other group. Student's t-test;

$\#, p<0.05$ in relation to the initial evaluation of the same group. One-Way ANOVA for repeated measures. 


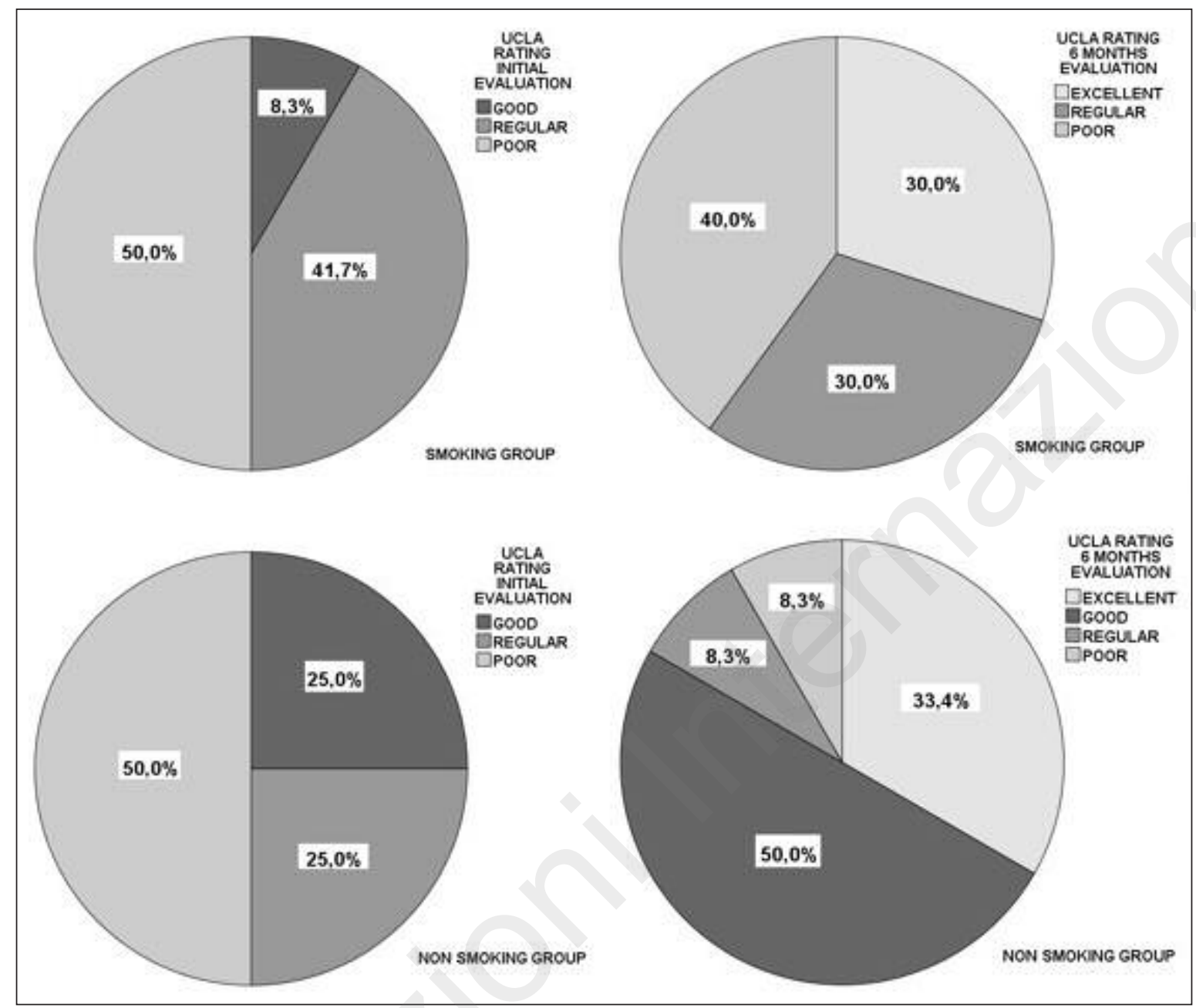

Figure 4. Classification of the initial UCLA scale and 6 months after intervention.

ty, the score decreased only in the 90-day evaluation $(p<0.05)$. Regarding total SPADI, the score decreased significantly only in the 90-day evaluation $(p<0.05)$ (Tab. IV).

Smokers started the treatment protocol with a significantly lower ROM of forward flexion, abduction, and external rotation in the left shoulder $(p<0.05)$ compared to nonsmokers. At the end of the treatment, smokers had less active bilateral shoulder flexion and less abduction of the left shoulder $(p<0.05)$. Only the nonsmoking group demonstrated a significant improvement in the range of motion regarding active bilateral forward flexion, abduction, extension, and external rotation of the right upper limb $(p<0.05)$ from the initial to the final evaluation. The right internal rotation improved in the nonsmoking group in the 90day evaluation $(p<0.05)$. The smoking group, in turn, improved only the right abduction in the 90-day evaluation $(p<0.05)($ Tab. V).

The smoking participants showed a significantly lower isometric force in the initial evaluation both for bilateral forward flexion and abduction and for external and internal rotation of the upper left shoulder $(p<0.05)$.
At the end of the intervention protocol, it was observed that smokers still showed a significantly lower muscle force for forward flexion, abduction, bilateral shoulder external rotation, and internal rotation of the left shoulder $(p<0.05)$. In the six-month evaluation, the nonsmoking group showed an isometric force greater than the smoking group for forward flexion, abduction, and internal rotation of the left shoulder $(p<0.05)(T a b . V I)$.

\section{Discussion}

In the present study, we sought to analyze the influence of smoking on subjects with shoulder rotator cuff tear submitted to a physical therapy protocol. To date, few or almost no studies have been found in the literature relating post-physical therapy functional outcomes with cigarette smoking.

By calculating the Pack-Years, we verified that the smoking group consumed cigarettes as a long-term habit. The time of cigarette consumption was $30.67 \pm$ 12.19 years, being $12.17 \pm 8.42$ cigarettes/day. The 


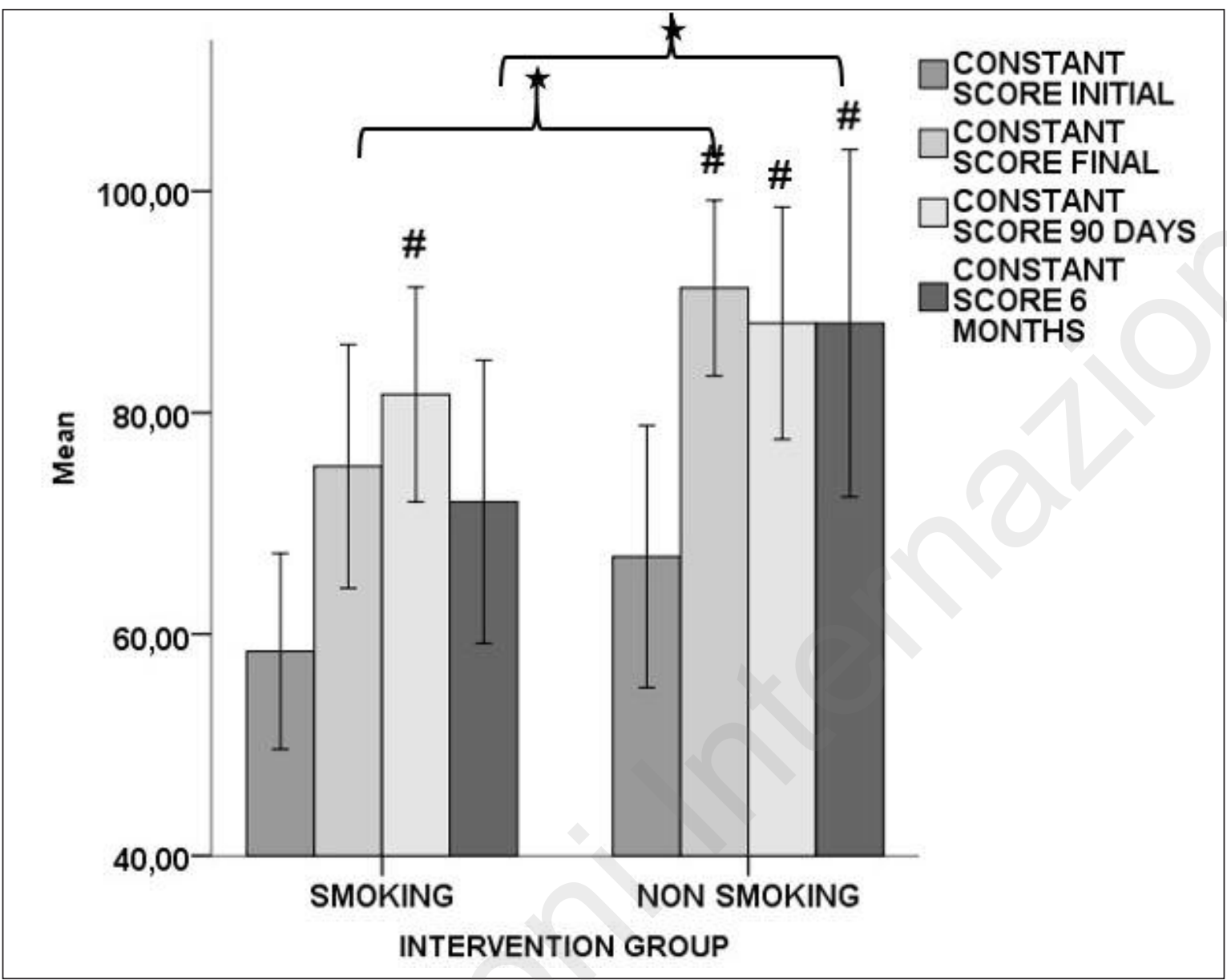

Figure 5. Score of the Constant-Murley questionnaire in the study groups.

Legend: *,$p<0.05$ in relation to the same evaluation of the other group. Student's t-test;

$\#, p<0.05$ in relation to the initial evaluation of the same group. One-Way ANOVA for repeated measures.

total calculation of the Pack-Years was $20.21 \pm$ 17.49. There are numerous studies in the literature demonstrating that smoking is associated with the development of musculoskeletal injuries ${ }^{4-6,8,13}$, and interferes with tissue repair processes, thus making these tissues more susceptible to injury ${ }^{5}$. The harmful effects have also been shown to persist for a period after smoking cessation ${ }^{5}$.

Lundgreen et al. ${ }^{4}$ evaluated the effect of smoking on supraspinatus tendon degeneration ${ }^{4}$. Samples of the supraspinatus tendon of 10 smokers and 15 nonsmokers with total thickness rupture were compared according to the histopathological evolution of the tendon ${ }^{4}$. Immunohistochemistry was used to assess the density of apoptotic cells and proliferation ${ }^{4}$. The extent of tendon degeneration was classified according to a revised version of the Bonar tendon histopathology score ${ }^{4}$. The supraspinatus tendons of smokers presented significantly more advanced degenerative changes and increased density of apoptotic cells, accompanied by a reduction of tenocyte intensity, and a positive regulation of proliferative activity ${ }^{4}$. These results demonstrated that smoking is associat- ed with worsened supraspinatus tendon histopathology and increased apoptosis, which may indicate a reduction in the healing capacity of the tendon in smokers ${ }^{4}$. We believe that this condition may be the main reason for both the arrival of these smokers to rehabilitation with more unfavorable functional conditions than nonsmokers and the less significant improvement in post-physical therapy recovery presented by smokers in this study.

Bishop et al. ${ }^{6}$ conducted a systematic review to investigate the influence of smoking on shoulder joint and rotator cuff tear. They concluded that smoking has a negative impact on shoulder health, being associated with a higher prevalence of tendon degeneration, increased RC tears, and increased rupture rates. Moreover, younger patients were also affected, thus increasing the need for surgical interventions.

Baumgarten et al. ${ }^{13}$ aimed to analyze the relationship between smoking and a greater risk of RC ruptures through a questionnaire applied to 586 patients over 18 years of age, without previous surgery, who had a diagnosis of shoulder disease through imaging examination ${ }^{13}$. They concluded that smoking was an im- 
Table IV. SPADI results in smokers and nonsmokers.

\begin{tabular}{|c|c|c|c|}
\hline Variable & Nonsmoking Group & Smoking Group & p value ${ }^{\$}$ \\
\hline \multicolumn{4}{|c|}{ SPADI Pain, points \pm sd } \\
\hline Initial & $68.13 \pm 24.48$ & $67.21 \pm 23.17$ & 0.93 \\
\hline Final & $23.13 \pm 17.33^{\#}$ & $35.83 \pm 36.66$ & 0.29 \\
\hline 90-day & $23.67 \pm 23.31^{\#}$ & $29.20 \pm 27.23^{\#}$ & 0.62 \\
\hline 6-month & $20.28 \pm 27.76^{\#}$ & $44.40 \pm 23.05^{\$}$ & 0.04 \\
\hline \multicolumn{4}{|c|}{ SPADI Disability, points \pm sd } \\
\hline Initial & $45.55 \pm 28.26$ & $51.25 \pm 22.13$ & 0.60 \\
\hline Final & $17.25 \pm 14.15^{\#}$ & $23.70 \pm 29.85$ & 0.51 \\
\hline 90-day & $13.71 \pm 15.48^{\#}$ & $11.87 \pm 15.39^{\#}$ & 0.77 \\
\hline 6-month & $11.95 \pm 19.19^{\#}$ & $26.71 \pm 20.43$ & 0.10 \\
\hline \multicolumn{4}{|c|}{ Total SPADI, points \pm sd } \\
\hline Initial & $56.07 \pm 24.60$ & $57.19 \pm 21.34$ & 0.91 \\
\hline Final & $20.39 \pm 16.02^{\#}$ & $28.91 \pm 31.82$ & 0.42 \\
\hline 90-day & $17.58 \pm 18.13^{\#}$ & $19.99 \pm 18.38^{\#}$ & 0.76 \\
\hline 6-month & $15.11 \pm 22.41^{\#}$ & $33.57 \pm 19.80$ & 0.05 \\
\hline
\end{tabular}

Student's t-test and Kruskal-Wallis test between the groups;

$\# p<0.05$ in relation to the initial evaluation of the same group. One-Way ANOVA for repeated measures.

portant risk factor for disease development, and one of the comorbidities that has negative effects on the different stages of shoulder pathologies ${ }^{13}$.

Among the numerous substances present in cigarettes are nicotine and carbon monoxide. These two substances decrease the microperfusion and oxygenation of tissues, leading to tissue hypoxia through vasoconstriction, thus altering the cellular metabolism and the potential for tendon recovery $4,8,17,18$. RC already presents a hypovascular area near the insertion of the supraspinatus tendon, with a $10-15 \mathrm{~mm}$ reduction in blood supply4,8,17,18, as initially described by Codman ${ }^{16}$. Our study demonstrated significantly better results in functional and quality of life scores through the UCLA, Constant, and SPADI-Brazil questionnaires in nonsmokers. Mallon et al. ${ }^{9}$, in a retrospective study, analyzed patients undergoing surgical treatment for $\mathrm{RC}$ rupture to test the hypothesis that nonsmokers present better postoperative scores than smokers. There were 95 smokers and 129 nonsmokers. The mean preoperative UCLA scores for smokers and nonsmokers were, respectively, 15.9 and 17.6 points. The mean postoperative scores were 25.0 and 32.0 points, respectively. Nonsmokers had a significantly higher increase in UCLA scores compared to smokers. Bishop et al. ${ }^{6}$ also found worse functional results in the UCLA questionnaire for smokers. Junior et al. ${ }^{18}$ evaluated the influence of smoking on surgical outcomes in the recovery from complete RC tears classified as C1 and C2 according to Snyder's classification ${ }^{19}$. One hundred and sixty-six patients who underwent surgical procedures, including smokers and nonsmokers, were evaluated according to the criteria of the World Health Organization (WHO) ${ }^{19}$. Of the 166 patients, 21 were smokers and 145 were nonsmokers ${ }^{19}$. Function was assessed using the UCLA criteria $^{19}$. Smokers had a final mean of 32.6 points, while nonsmokers presented a final mean of 33.8 points ${ }^{19}$. The Authors concluded that smoking interferes with the outcome of the recovery from small and medium rotator cuff tears ${ }^{19}$. Our findings also demonstrate worse functional outcomes in the UCLA scale for smokers at the end of the intervention. Smokers reached $25.92 \pm 5.18$ points, while nonsmokers had $30.33 \pm 3.03$ points. The same difference was observed in the 180-day evaluation, where smokers presented a mean value of $21.00 \pm 8.80$ points, and nonsmokers presented $28.38 \pm 9.05$ points. 
Table V. Active range of motion (ROM) of the shoulder in smokers and nonsmokers.

\begin{tabular}{l} 
Variable \\
\hline Right active flexion, degrees \pm sd \\
Initial \\
Final \\
90-day \\
6-month \\
Left active flexion, degrees \pm sd \\
Initial \\
Final \\
90-day \\
6-month
\end{tabular}

Right active abduction, degrees \pm sd

Initial
Final
90-day
6-month

Left active abduction, degrees $\pm \mathrm{sd}$

Initial
Final
90-day
6 -month

Right active extension, degrees \pm sd

Initial
Final
90-day
6-month

Left active extension, degrees \pm sd

Initial
Final
90-day
6-month

Right active external rotation, degrees $\pm \mathrm{sd}$

Initial
Final
90-day
6 -month

Left active external rotation, degrees \pm sd

$\begin{array}{ll}\text { Initial } & 77.83 \pm 15.03 \\ \text { Final } & 76.92 \pm 11.86 \\ \text { 90-day } & 74.08 \pm 11.04 \\ \text { 6-month } & 75.00 \pm 15.84\end{array}$

Smoking Group

$p$ value

$\begin{array}{ll}134.50 \pm 28.50 & 0.94 \\ 145.75 \pm 32.03 & 0.05 \\ 153.20 \pm 25.32 & 0.66 \\ 159.00 \pm 15.67 & 0.98\end{array}$

$166.42 \pm 12.97$

$139.83 \pm 35.92$

0.03

$173.58 \pm 8.08^{\#}$

$160.42 \pm 10.97$

0.00

$170.25 \pm 7.17$

$161.58 \pm 36.41$

$159.10 \pm 12.56$

0.03

$155.80 \pm 32.22$

0.70

$124.08 \pm 25.93$

$154.33 \pm 19.68^{\#}$

$149.92 \pm 31.38^{\#}$

$155.50 \pm 36.55^{\#}$

$127.50 \pm 31.21$

0.77

$144.00 \pm 26.36$

0.29

$149.20 \pm 17.84^{\#}$

0.95

$143.10 \pm 32.38$

0.41

$154.42 \pm 20.04$

$124.92 \pm 37.41$

0.03

$165.83 \pm 9.81$

$165.33 \pm 7.50$

$161.58 \pm 36.40$

$141.75 \pm 26.85$

0.01

$155.50 \pm 14.71$

0.05

$151.00 \pm 30.69$

0.73

$48.00 \pm 11.26$

$52.50 \pm 10.89$

0.33

$55.92 \pm 4.77^{\#}$

$57.08 \pm 5.02^{\#}$

$53.58 \pm 8.21$

0.41

$55.10 \pm 8.25$

0.52

$55.40 \pm 6.87$

0.96

$55.42 \pm 6.78$

$50.33 \pm 13.37$

0.26

$59.17 \pm 3.01$

$58.58 \pm 2.57$

$55.58 \pm 15.01$

$55.92 \pm 5.30$

0.08

$52.70 \pm 9.33$

0.08

$55.90 \pm 5.21$

0.95

$66.17 \pm 11.87$

$67.92 \pm 17.42$

0.77

$75.67 \pm 10.47^{\#}$

$77.50 \pm 8.73$

0.65

$70.50 \pm 13.59$

$74.80 \pm 8.69$

0.38

$75.20 \pm 12.37$

0.77

$66.58 \pm 25.84$

$62.67 \pm 20.68$

0.05

$77.83 \pm 12.05$

0.85

$71.60 \pm 6.87$

0.53

$71.40 \pm 9.75$

0.52 
Continue from Table $\mathbf{V}$.

\begin{tabular}{lcccc}
\hline Right active internal rotation, degrees \pm sd & & & & \\
Initial & $76.75 \pm 18.32$ & $76.33 \pm 17.07$ & 0.95 \\
Final & $86.00 \pm 4.63$ & $89.33 \pm 2.31$ & 0.04 \\
90-day & $82.67 \pm 10.25^{\#}$ & $79.20 \pm 16.44$ & 0.57 \\
6-month & $79.75 \pm 25.43$ & $81.70 \pm 8.22$ & 0.81 \\
Left active internal rotation, degrees $\pm s d$ & & & 0.08 \\
Initial & $88.33 \pm 8.45$ & $75.67 \pm 21.26$ & 0.25 \\
Final & $89.25 \pm 3.11$ & $87.25 \pm 4.88$ & 0.10 \\
90-day & $89.00 \pm 2.34$ & $79.50 \pm 16.55$ & 0.82 \\
6-month & $83.83 \pm 15.05$ & $85.00 \pm 8.01$ & 0 \\
\hline
\end{tabular}

Student's t-test and Kruskal-Wallis test between the groups;

$\# p<0.05$ in relation to the initial evaluation of the same group. One-Way ANOVA for repeated measures;

$p<0.05$ in relation to the final evaluation of the same group. ANOVA One-Way ANOVA for repeated measures.

Kukkonen et al. ${ }^{11}$ analyzed five hundred seventy-six consecutive shoulders with $\mathrm{RC}$ tear to evaluate the effect of smoking on preoperative, intraoperative, and postoperative RC recovery ${ }^{11}$. Functional analysis was performed using the Constant score, where smokers achieved 71 points, and nonsmokers obtained 75 points ${ }^{11}$. Our study also showed differences in this score, where smokers had a final score of $77.33 \pm$ 14.90 points, while nonsmokers had $91.25 \pm 12.49$ points.

Bishop et al. ${ }^{6}$ verified that women with a history of smoking for a period of 10 to 20 years present a greater risk of bilateral shoulder pain. In our study, the prevalence of tears occurred in women $(n=15)$. There were four bilateral tears in the smoking group, and only two in the nonsmoking group.

Kane et al. ${ }^{20}$ performed a subjective shoulder evaluation through an online survey of 166 subjects to relate lifestyle and shoulder dysfunction ${ }^{20}$. They used the Oxford questionnaire, in addition to a shoulder assessment questionnaire, and a subjective shoulder evaluation system ${ }^{20}$. Three of the questions related to shoulder pain revealed statistically significant differences between smokers and nonsmokers, where smokers reported complaints of major pain ${ }^{20}$. Our study assessed the pain of subjects through the Visual Analogue Pain Scale (VAS). There was a significant reduction of pain after the intervention protocol in both smokers and nonsmokers. Notwithstanding, in the 90- and 180-day evaluations, only smokers had pain levels similar to the baseline. Nonsmokers reported levels of pain reduction even six months after the intervention protocol. Kane et al. ${ }^{20}$ also verified that, in addition to the shoulder function being lower in smokers, they also presented a lower bilateral active range of motion (ROM) of the shoulder ${ }^{20}$. The outcome suggested by the Authors is a quantifiable relationship between smoking, shoulder dysfunction, and pain ${ }^{20}$. In our study, smokers presented lower bilateral active shoulder flexion and less abduction of the left shoulder $(p<0.05)$ at the end of treatment. In the initial evaluation, smokers already had a greater limitation in the active and passive forward flexion, abduction, and external rotation of the left shoulder $(p<0.05)$. Nonsmokers showed a decrease in the left active ROM of forward flexion in the six-month evaluation. However, this group showed a prevalence of $\mathrm{RC}$ tears in the right shoulder, which may have contributed to this result.

In their review article, Abate et al. ${ }^{21}$ found that loss of muscle mass and muscle strength is related to the individual's age and is significantly influenced by cigarette smoking ${ }^{21}$. Musculoskeletal damage induced by cigarette smoking is due to impaired muscle metabolism, increased inflammation, and oxidative stress ${ }^{20}$.

In a cross-sectional study, Saito et al. ${ }^{22}$ investigated the link between smoking and muscle strength in 4,249 Japanese men. To assess muscle strength, a suitable dynamometer was used for each measure 22 . The amount of cigarette consumption was expressed by the Brinkman index, which is the number of cigarettes smoked per day multiplied by the number of years of smoking ${ }^{22}$. A Brinkman index greater than or equal to 400 identifies subjects as heavy smokers, and lower than 400 as moderate smokers ${ }^{22}$. Smokers with a Brinkman index greater than 400 had lower muscle strength results ${ }^{21}$. The Authors concluded that smoking may be negatively associated with the individual's overall muscle strength ${ }^{22}$. In our study, we found that smokers arrived at the initial evaluation with a greater deficit of muscle strength compared to nonsmokers for forward flexion and bilateral abduction, external rotation, and left internal rotation. At the end of the intervention protocol, smokers still showed lower muscle strength for forward flexion, abduction, and bilateral external rotation of the shoulder $(p<0.05)$ compared to nonsmokers, which demonstrates the influence of cigarette smoking on muscle strength, symptomatology, mobility, and function in this population. 
Table VI. Shoulder muscle strength (MVIC) in smokers and nonsmokers.

\begin{tabular}{|c|c|c|c|}
\hline Variable & Nonsmoking Group & Smoking Group & $p$ value \\
\hline \multicolumn{4}{|c|}{ Right flexion, $\mathrm{kg} \pm \mathrm{sd}$} \\
\hline Initial & $8.83 \pm 2.69$ & $6.83 \pm 1.75$ & 0.04 \\
\hline Final & $11.42 \pm 2.84^{\#}$ & $7.25 \pm 3.33$ & 0.00 \\
\hline 90-day & $10.33 \pm 2.64$ & $8.80 \pm 2.70$ & 0.20 \\
\hline 6-month & $10.17 \pm 4.61$ & $7.60 \pm 2.99$ & 0.13 \\
\hline \multicolumn{4}{|l|}{ Left flexion, $\mathrm{kg} \pm \mathrm{sd}$} \\
\hline Initial & $9.75 \pm 2.01$ & $6.83 \pm 2.13$ & 0.00 \\
\hline Final & $10.50 \pm 2.24$ & $7.75 \pm 3.28$ & 0.03 \\
\hline 90-day & $10.92 \pm 3.40$ & $8.40 \pm 2.63$ & 0.06 \\
\hline 6-month & $10.08 \pm 3.55$ & $7.10 \pm 2.96$ & 0.04 \\
\hline \multicolumn{4}{|c|}{ Right Abduction, $\mathrm{kg} \pm \mathrm{sd}$} \\
\hline Initial & $11.08 \pm 2.43$ & $9.17 \pm 1.99$ & 0.05 \\
\hline Final & $13.42 \pm 2.61$ & $9.92 \pm 3.00$ & 0.00 \\
\hline 90-day & $12.75 \pm 2.83$ & $10.30 \pm 4.03$ & 0.56 \\
\hline 6-month & $11.83 \pm 4.59$ & $9.00 \pm 3.43$ & 0.11 \\
\hline \multicolumn{4}{|c|}{ Left Abduction, $\mathrm{kg} \pm \mathrm{sd}$} \\
\hline Initial & $11.92 \pm 2.94$ & $8.92 \pm 2.64$ & 0.01 \\
\hline Final & $12.83 \pm 2.59$ & $9.17 \pm 2.66$ & 0.00 \\
\hline 90-day & $12.42 \pm 2.84$ & $10.50 \pm 2.88$ & 0.13 \\
\hline 6-month & $11.83 \pm 3.69$ & $9.00 \pm 3.59$ & 0.00 \\
\hline \multicolumn{4}{|c|}{ Right external rotation, $\mathrm{kg} \pm \mathrm{sd}$} \\
\hline Initial & $9.00 \pm 2.41$ & $8.50 \pm 2.75$ & 0.64 \\
\hline Final & $11.50 \pm 3.53$ & $8.00 \pm 3.07$ & 0.02 \\
\hline 90-day & $11.17 \pm 3.24$ & $9.10 \pm 2.68$ & 0.12 \\
\hline 6-month & $10.92 \pm 4.96$ & $8.50 \pm 3.10$ & 0.18 \\
\hline \multicolumn{4}{|c|}{ Left external rotation, $\mathrm{kg} \pm \mathrm{sd}$} \\
\hline Initial & $11.58 \pm 3.34$ & $8.83 \pm 3.13$ & 0.05 \\
\hline Final & $13.50 \pm 3.06$ & $9.08 \pm 2.97$ & 0.00 \\
\hline 90-day & $12.75 \pm 2.66$ & $10.60 \pm 4.14$ & 0.18 \\
\hline 6-month & $11.92 \pm 4.62$ & $8.30 \pm 3.53$ & 0.18 \\
\hline \multicolumn{4}{|c|}{ Right internal rotation, $\mathrm{kg} \pm \mathrm{sd}$} \\
\hline Initial & $10.83 \pm 2.79$ & $9.50 \pm 3.15$ & 0.28 \\
\hline Final & $13.25 \pm 4.14$ & $10.50 \pm 3.83$ & 0.10 \\
\hline 90-day & $11.66 \pm 4.01$ & $11.40 \pm 4.22$ & 0.88 \\
\hline 6-month & $12.08 \pm 5.35$ & $9.80 \pm 3.88$ & 0.26 \\
\hline \multicolumn{4}{|c|}{ Left internal rotation, $\mathrm{kg} \pm \mathrm{sd}$} \\
\hline Initial & $13.58 \pm 3.85$ & $9.67 \pm 3.82$ & 0.02 \\
\hline Final & $14.67 \pm 3.37$ & $10.50 \pm 4.23$ & 0.01 \\
\hline 90-day & $13.75 \pm 3.79$ & $11.50 \pm 5.15$ & 0.27 \\
\hline 6-month & $13.08 \pm 5.50$ & $10.00 \pm 5.29$ & 0.02 \\
\hline
\end{tabular}

Student's t-test and Kruskal-Wallis test between the groups;

$\# p<0.05$ in relation to the initial evaluation of the same group. One-Way ANOVA for repeated measures; $p<0.05$ in relation to the final evaluation of the same group. One-Way ANOVA for repeated measures.

\section{Study limitations}

To date, there are almost no studies relating postphysical therapy functional outcomes with cigarette smoking. We consider this study as an important predictor of the response of these participants to a conservative rehabilitation program in $\mathrm{RC}$ tear. The sam- ple size and the short follow-up period can be considered a limiting factor in the extrapolation of the obtained results. To confirm these findings, further studies relating smoking and physical rehabilitation should be performed with larger samples and a larger follow-up. 


\section{Conclusion}

- Smokers arrive at the physical therapy service with significantly greater functional deficits in shoulder RC injuries compared to nonsmokers.

- Cigarette smoking worsens functional outcomes following a physical therapy program for shoulder $\mathrm{RC}$ injuries.

- Nonsmokers significantly reduced pain level and improved long-term mobility and function following a shoulder rehabilitation protocol when compared to smokers.

\section{Conflict of interest}

The Authors declare no conflict of interest.

\section{References}

1. Whittle S, Buchbinder R. In the Clinic Rotator Cuff Disease. Ann Intern Med. 2015;162(1):1-15.

2. Metzker BAC. Tratamento conservador na síndrome do impacto do ombro. Fisioter Mov. 2010;23(1):141-151.

3. Peinado TD, Stefanutto AS. Cinesioterapia para síndrome do impacto. Anuário da produção de iniciação científica discente. 2011;14(26):289-297.

4. Lundgreen K, Lian OB, Scott A, Nassab P, Fearon A, Engebretsen L. Rotator Cuff Tear Degeneration and Cell Apoptosis in Smokers vs Nonsmokers. Arthroscopy. 2014;30(8):936941.

5. Altarac M, Gardner JW, Popovich RM, Potter R, Knapik JJ, Jones BH. Cigarette Smoking and Exercise-Related Injuries among Young Men and Women. Am J Prev Med. 2000;18(3): 96-102.

6. Bishop JY, Santiago-Torres JE, Rimmke N, Flanigan DC. Smoking Predisposes to Rotator Cuff Pathology and Shoulder Dysfunction: A Systematic Review. Arthroscopy. 2015;31 (8):1-8.

7. Barbosa RI, Goes R, Mazzer N, Fonseca MCR. A influência da mobilização articular nas tendinopatias dos músculos bíceps braquial e supra-espinal. Braz J Phys Ther. 2008;12(4):298-303.

8. Carbone S, Gumina S, Arceri V, Campagna V, Fagnani F,
Postacchini F. The impact of preoperative smoking habit on rotador cuff tear: cigarrette smoking influences rotador cuff tear sizes. J shoulder Elbow Surg. 2012;21(1):56-60.

9. Mallon WJ, Misamore G, Snead DS, Denton P. The impact of preoperative smoking habits on the results of rotator cuff repair. J Shoulder Elbow Surg. 2004;13(2):129-132.

10. Almeida A, Valin MR, Zampieri R, Almeida NC, Roveda G, Agostini AP. Análise comparativa do resultado da sutura artroscópica da lesão do manguito rotador em pacientes fumantes e não fumantes. Rev Bras Ortop. 2011;46(2):172-175.

11. Kukkonen J, Kauko T, Virolainen $P$, Äärimaa V. Smoking and operative treatment of rotador cuff tear. Scand J Med Sci Sports. 2014;24(2):400-403.

12. Lee JJ, Patel R, Biermann JS, Dougherty PJ. The Musculoskeletal Effects of Cigarette Smoking. J Bone Joint Surg Am. 2013;95(9):850-859.

13. Baumgarten KM, Gerlach D, Galatz LM, et al. Cigarrete smoking increases the risk for rotador cuff tears. Clin Orthop Relat Res. 2010;468(6):1534-1541.

14. Santiago-Torres J, Flanigan DC, Butler RB, Bispo JY. The effect of smoking on rotator cuff and glenoid labrum surgery: A systematic review. Am J Sports Med. 2015; 43(3): 745-751.

15. Padulo J, Oliva F, Frizziero A, Maffulli N. Muscles, Ligaments and Tendons Journal - Basic principles and recommendations in clinical and field science research: 2016 update. MLTJ. 2016;6(1):1-5.

16. Factor D, Dale B. Current concepts of rotador cuff tendinopathy. In J Sports Ther. 2014; 9(2): 274-281.

17. Via AG, Cupis M, Spoliti M, Oliva F. Clinical and biological aspects of rotator cuff tears. MLTJ. 2013; 3(2): 70-79.

18. Sambandam SN, Khanna V, Gul A, Mounasamy V. Rotator cuff tears: An evidence based approach. World J Orthop. 2015; 6(11): 902-918.

19. Júnior SCP, Luciano LG, Zotto CD, et al. Resultado da reparação do manguito rotador em lesões do tipo $\mathrm{C} 1$ e $\mathrm{C} 2$ de Snyder, considerando fumantes e não fumantes. Rev Bras Ortop. 2010; 45(6): 554-556.

20. Kane S, Conus S, Haltom D, Hirshorn K, Pak Y, Vigdorchik J. A Shoulder Health Survey. Correlating Behaviors and Comorbidities with Shoulder Problems. Sports Health. 2010; 2(2): 119-134.

21. Abate M, Vanni D, Pantalone A, Saline V. Cigarrete smoking and musculoskeletal disorders. Muscles Ligaments Tendons J. 2013; 3(2): 63-68.

22. Saito T, Miyatake N, Sakano $\mathrm{N}$ et al. Relationship between Cigarette Smoking and Muscle Strength in Japanese Men. J Prev Med Public Health. 2012; 45(6): 381-386. 